\title{
REVIEW \\ Summary of the Frontier Introduction of Preparation of Secondary Metabolites in Plant Cell Culture
}

\author{
Qiaole $\mathrm{Li}^{1^{*}}{\text { Tengfei } \mathrm{He}^{2} \text { Haoran Niu }}^{1}$ \\ 1. Biotechnology, North China University of Science and Technology, Tangshan, Hebei, 063000, China \\ 2. Material Processing and Control Engineering, North China University of Science and Technology, Hebei, 063000, \\ China
}

\section{ARTICLE INFO}

Article history

Received: 31 July 2020

Accepted: 31 July 2020

Published Online: 30 September 2020

Keywords:

Plant cell culture

Secondary metabolites

Plants

Applications

\begin{abstract}
Plant cell culture technology is a technology that applies the research results of cell engineering to produce plant biological products at the cellular level. In recent years, the secondary metabolites of plants have attracted more and more attention. The use of plant cell culture technology is a fast and efficient method of producing secondary metabolites.
\end{abstract}

development prospects are not satisfactory. Therefore, the use of plant cell culture to produce secondary metabolites has become an important way to solve the problem.

\section{Introduction to the Preparation of Second- ary Metabolites from Plant Cells}

Secondary metabolites refer to a large class of small molecular organic compounds that are not necessary for cell life activities or normal plant growth and development in plants. Compared with other methods, the use of plant cell culture technology to prepare secondary metabolites has great advantages:

(1)Under the conditions of complete manual control,

*Corresponding Author:

Qiaole Li,

Biotechnology, North China University of Science and Technology, No. 21 Bohai Avenue, Caofeidian District, Tangshan, Hebei, 063000, China;

Email:1446087491@qq.com 
continuous production can be achieved, regardless of region and season, saving land and facilitating industrial production;

(2)Obtain metabolites that exceed the yield of the original plant by changing the culture conditions and selecting superior lines;

(3)Plant cell culture technology is completed under aseptic conditions, which can eliminate the intrusion of germs and insect pests on medicinal plants;

(4)Genetic manipulation of the synthetic route of active ingredients can increase the production of required secondary metabolites on a large scale;

(5)Plant cell culture technology is also an important way to solve resource problems.

After a long period of development, plant cell culture technology has established a standard operating procedure.

\subsection{Selection of Highly Expressing Cell Line}

Screening high-yield and stable cell lines can greatly increase the yield and quality of secondary metabolites. At present, the stable expression system and transient expression system in transgenic technology ${ }^{[2]}$ are used to produce high-yielding cell lines with the target gene. After a large number of screenings, cell lines that can produce specific secondary metabolites can be obtained.

Transgenic technology produces cell lines with low investment, low cost and safe use. The plant cell culture conditions are simple, easy to survive, and easy to operate. The main methods used are: Agrobacterium-mediated method, recombinant virus infection of plants, and gene gun transformation method And pollen tube channel transformation method ${ }^{[3]}$.

\subsection{Suitable Environmental Conditions}

When different plants produce secondary metabolites, the required environmental conditions are different. When plant cell culture is carried out, the medium components such as carbon source, nitrogen source, organic matter, exogenous plant hormones, plant growth regulators, inorganic The requirements for salt and $\mathrm{pH}$ are different. At the same time, proper light and temperature are important factors to increase the production of secondary metabolites of plant cells. For example, when roselle suspension cells synthesize anthocyanins, blue light is the most effective monochromatic light that promotes the production of anthocyanins by roselle cells. The output is $416 \mathrm{mg} / \mathrm{L}$, which is similar to panchromatic light. Red light and orange light are ineffective. Other monochromatic light As its wavelength approaches blue light, the positive effect increases ${ }^{[4]}$.

\subsection{Production of Secondary Metabolites}

(1) Precursor feeding and biotransformation, and insufficient precursor compounds will affect the anabolism of secondary metabolites in plants. Reactions such as saponification, esterification, demethylation and double bond reduction can occur in plants. For example, adding farnesol to Tripterygium wilfordii cell culture can increase the production of tripterygium hydroxylactone by more than 3 times. The addition of L phenylalanine can increase the yield of shikonin by 3 times $^{[4]}$. The type, concentration and time of the added precursor will also affect the preparation of secondary metabolites.

(2) Cell fixation system, the cell fixation system can maintain the amount of cells in the reaction tank, immobilization makes the reaction activity stable, and can be produced continuously for a long time, and is easy to separate from the cells as the catalyst ${ }^{[5]}$, At the same time, it is easy to control the environmental conditions and substrate concentration in production.

However, when the fixed system is used for plant cell culture, the cell culture density is high, the supply and delivery of oxygen and nutrients are a difficult problem, resulting in relatively slow growth of cultured plant cells, and even inhibiting cell growth and development, cell genetic stability, and non-secretion. Form release of secondary metabolites and other issues, these issues still need us to further study.

(3)The two-phase culture method prevents the metabolic effects of secondary metabolites from inhibiting their biosynthesis and protects them from the influence of enzymes or acids in the medium. For example, only $1 \%$ of the thiophene synthesized by the hairy roots of maidenhair can be secreted into the culture medium. Adding hexadecane to the culture system can promote the secretion of $30 \%$ to $60 \%$ of thiophene ${ }^{[6]}$.

(4) The two-step culture method better solves the contradiction between the growth of cell biomass and the accumulation of secondary metabolite products, and greatly improves the yield of the target product ${ }^{[7]}$. Coptidis cells were cultured in the culture medium for 3 weeks, and then cultured in the production medium for another 3 weeks. Each liter of culture solution can obtain $556 \mathrm{mg}$ of alkaloids, and the yield is 1.72 times that of the one-step culture method.

(5) The use of inhibitors and antisense technology. Antisense technology is the use of modern molecular biology technology to introduce artificially synthesized antisense RNA into the plant genome, and combine with the key enzyme gene RNA of the metabolic pathway to be inhibited to form double-stranded RNA to block 
the normal expression of genes, thereby promoting The expression of the target gene. Nowadays, antisense technology is more and more widely used in the production of plant secondary metabolites. For example, after the antisense RNA of ethylene synthase in tomato is transferred into tomato, ethylene synthesis is reduced by $97 \%{ }^{[8]}$.

(6) Application of elicitor. Elicitors can enhance the respiration of cells, provide the energy required to produce secondary metabolites, change cell structure, and facilitate the formation, transportation and accumulation of secondary metabolites. The elicitors used in plant cell culture mainly include glycoprotein elicitors, protein elicitors, polysaccharide elicitors, and microbial elicitors ${ }^{[9]}$.Among them, the factors that affect the elicitor induction effect include the type, concentration and inoculation time of the elicitor.

\section{Summary and Outlook}

Although compared with other methods, plant cell culture technology has obvious advantages, but there are still many problems that need to be solved to truly realize the large-scale industrialization of plant cell culture. With the development of molecular biology, it will help plant cell culture to solve many theoretical and practical problems.

\section{References}

[1] Xianghai Kong. Research Progress in Cell Culture Technology of Plant Secondary Metabolites[A]. Journal of Longying University, 2005(12).
[2] Shuying Gao, Aiguang Guo, Weibo Jin, etc. Research progress in the production of medicinal protein by transgenic technology[J]. Northwestern Journal of Botany, 2003, 23(6): 1044-1048.

[3] Zhenquan Yang, Qiaoquan Liu, Xinan Jiao. Using transgenic plants to express medicinal proteins $[\mathrm{J}]$, Chinese Journal of Bioengineering, 2004, 24(3): 2224.

[4] Ya Gao, Lijie Yu. Research Progress of Plant Cell Culture Technology to Produce Secondary Metabolites[A]. Journal of Mudanjiang Teachers College (Natural Science Edition), 2008(1): 25-27.

[5] Tangbing Cui, Yong Guo, Weitie Lin. Methods to increase the production of stimulating metabolites produced by plant cell culture[J]. Plant Physiology Communications, 2001, 37(5): 479-482.

[6] Chunqiao Xiao, Huaxiang Zhang, Hong Gao, etc. Several ways to promote plant cell culture to produce secondary metabolites[J]. Journal of Wuhan Institute of Chemical Technology, 2005, 27(1): 28-30.

[7] Yufang Ma, Jihong Xu. Methods to increase the yield of secondary metabolites in plant cell culture $[\mathrm{J}]$. Journal of Yunnan University: Natural Science Edition, 2003, 25 (Supplement): 142-145.

[8] Kai Hu, Feng Tan. Large-scale cultivation technology of medicinal plant cells[J]. Plant Physiology Communications, 2004, 40(2): 251-257.

[9] Linghua Zhang, Yong Guo. Selection and cultivation of medicinal plant cells to produce secondary metabolites[J]. Biotechnology Communications, 2006, 17(1): 105-108 